\title{
Análise morfométrica dos elementos traqueais em quatro espécies de Portulaca (Portulacaceae)
}

\author{
José Hernandes-Lopes ${ }^{1,2}$ e Gladys Flavia Melo-de-Pinna ${ }^{1}$
}

Recebido em 30/03/2007. Aceito em 22/08/2007

\begin{abstract}
RESUMO - (Análise morfométrica dos elementos traqueais em quatro espécies de Portulaca (Portulacaceae)). Este trabalho aborda aspectos da caracterização morfológica dos elementos traqueais dos órgãos vegetativos de quatro espécies de Portulacaceae (Portulaca hirsutissima Camb., P. halimoides L., P. werdermannii Poelln., e de P. mucronata Link.) ocorrentes na região Sudeste e Nordeste do Brasil. Os elementos de vaso observados são todos pequenos $(<25 \mu \mathrm{m})$ e com placa de perfuração simples. O padrão de espessamento da parede secundária varia de pontoado (mais comum em raízes) a escalariforme e helicoidal (mais comuns em caules e folhas). Testes estatísticos apontam para uma diferença significativa do diâmetro dos elementos de vaso entre os diferentes órgãos, sendo maior em raiz. Traqueídes foram observadas apenas em folhas de $P$. hirsutissima e $P$. werdermannii, semelhantes, morfologicamente, às traqueídes terminais ou aos idioblastos traqueoidais muito freqüentemente associados com as xerófitas. Características pedomórficas (juvenilismo) observadas neste trabalho podem, em parte, estar relacionadas com o transporte e estocagem de água como descrito em Cactaceae.
\end{abstract}

Palavras-chave: elementos traqueais, pedomorfose, Portulaca, Portulacaceae, Caryophyllales

\begin{abstract}
Morphometric analysis of tracheary elements in four Portulaca species). This paper deals with the morphological features of the tracheary elements of the vegetative organs in four Portulaca species (Portulaca hirsutissima Camb., P. halimoides L., $P$. wedermannii Poelln. and P. mucronata Link.) occurring in Southeast and Northeast Brazil. The vessel elements are small $(<25 \mu \mathrm{m})$ and with simple perforation plate. The pattern of wall thickening varied from bordered pitting (in roots) to scalariform and helicoidal (stem and leaves). Statistical methods show variation in vessel-element diameter in different vegetative organs; wider elements were observed in roots. Tracheids occurring in leaves of P. hirsutissima and P.wedermannii, have morphological features that are similar to terminal tracheids or tracheoid idiolasts frequently associated with xerophytes. The paedomorphic features (juvenlism) observed here may be related, in part, to aspects of water transport and storage as described in Cactaceae.
\end{abstract}

Key words: tracheary elements, paedomorphosis, Portulaca, Portulacaceae, Caryophyllales

\section{Introdução}

A família Portulacaceae é constituída por plantas de hábito herbáceo e arbustivo, muitas vezes suculentas e freqüentemente contendo mucilagem nas células parenquimáticas do caule e das folhas (Metcalfe \& Chalk 1985). Portulacaceae tem distribuição cosmopolita, incluindo cerca de 30 gêneros e 400 espécies, sendo ocorrentes no Brasil aproximadamente 30 espécies, pertencentes aos gêneros Portulaca e Talinum (Souza \& Lorenzi 2005).

Gibson (1994) menciona que uma potencial fonte de dados para análises filogenéticas em Caryophyllales é a anatomia do sistema vascular devido a sua grande quantidade de apomorfias peculiares. De acordo com o autor, a importância de se estudar os tecidos vasculares reside na necessidade de se encontrar sinapomorfias que esclareçam as relações filogenéticas, além de se estabelecer quais seriam as características esperadas para o ancestral da ordem (Gibson 1994). Dentre as famílias reconhecidas na ordem Caryophyllales, Cactaceae é a mais bem estudada quanto aos elementos traqueais, sendo registrada por alguns autores a ocorrência de um fenômeno conhecido como pedomorfose (Carlquist 1962; Gibson 1978; Arruda et al. 2004). Segundo Carlquist (1962), são encontrados elementos de vaso do xilema secundário com características ontogenéticas iniciais do metaxilema, como espessamento de parede secundária do tipo reticulado ou escalariforme. Essas características são registradas em órgãos com elevado grau de especialização ao acúmulo de água (suculência), que na maioria das espécies de Cactaceae é representado pelo caule (Gibson 1978, Arruda et al. 2004).

Neste trabalho são apresentados dados morfométricos dos elementos traqueais de quatro espécies de Portulaca, procurando caracterizar a pedomorfose em representantes de Portulacaceae, que apresentam caule e folha suculentos, como novo registro para a família.

\footnotetext{
1 Universidade de São Paulo, Instituto de Biociências, Departamento de Botânica, Rua do Matão 277, Travessa 14, C. Postal 11461, 05422-970 São Paulo, SP, Brasil

2 Autor para correspondência: zehernandes@gmail.com
} 


\section{Materiais e métodos}

Indivíduos de Portulaca hirsutissima Camb., P. halimoides L., P. werdermannii Poelln., e de P. mucronata Link. foram coletados nas regiões Sudeste e Nordeste do Brasil, mais especificamente nos estados de Minas Gerais, na região de Grão-Mogol (16 33 'S $42^{\circ} 53^{\prime} \mathrm{W}$ - P. mucronata, $16^{\circ} 35^{\prime} \mathrm{S}-42^{\circ} 54^{\prime} \mathrm{W}$ $P$. hirsutissima e $P$. halimoides) e Bahia, na cidade de

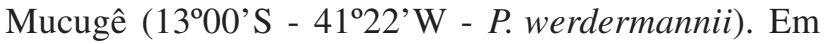
todas as localidades, a vegetação é caracterizada como campos rupestres apresentando solo pedregoso e apenas no caso de $P$. werdermannii houve coleta de indivíduos ocorrendo em bancos de areia. Parte do material foi utilizado na confecção de exsicatas que foram depositadas no Herbário da Universidade de São Paulo: P. hirsutissima (Hernandes-Lopes 01), P. mucronata (Hernandes-Lopes 02), P. halimoides (Hernandes-Lopes 06) e P. werdermannii (Hernandes-Lopes 10).

Amostras de raiz, caule e folha das quatro espécies foram fixadas em FAA 50\% (formaldeído, ácido acético glacial e etanol 50\%), sendo, após 48 horas, armazenadas em etanol 70\% (Johansen 1940).

Em caráter de pré-análise, para verificar possível diferença significativa entre o diâmetro radial e o diâmetro tangencial dos elementos de vaso, foram montadas lâminas de secções transversais, a mão livre, do caule e da raiz de cada espécie. Segundo IAWA Committee (1989), o diâmetro dos elementos que deve ser usado para medições é o tangencial.

Três indivíduos de cada espécie foram utilizados para a realização do presente estudo. Regiões mais desenvolvidas do caule e da raiz, com crescimento secundário estabelecido, assim como folhas completamente desenvolvidas, de cada indivíduo foram submetidas à solução de Franklin (1945 - peróxido de hidrogênio e ácido acético, 1:1) por 48 horas em estufa a $60^{\circ} \mathrm{C}$. Após este período as amostras foram lavadas com água destilada até a completa remoção da solução e armazenadas em etanol $70 \%$.

Para as observações qualitativas e quantitativas foram confeccionadas cinco lâminas/órgão/indivíduo. Excepcionalmente foi necessária a confecção de mais lâminas de folhas para se obter um $\mathrm{N}$ amostral satisfatório para cada indivíduo, devido ao pequeno número de elementos de vaso presente neste órgão. O material foi macerado sobre lâmina histológica, corado com safranina $1 \%$ e montado em glicerina 50\% (Purvis et al. 1964).

A escolha dos elementos traqueais a serem observados foi realizada ao acaso, com o intuito de não viciar a amostra, tendo-se apenas o cuidado de não incluir os elementos de vaso integrantes do protoxilema. Para a avaliação qualitativa foi observado um total de cem elementos de vaso/órgão/espécie, registrando os seguintes parâmetros: tipo de placa de perfuração, tipo de espessamento da parede secundária, posição da placa de perfuração (oblíqua ou transversa), localização da placa de perfuração (parede terminal ou lateral da célula) e presença e forma dos apêndices.

A análise quantitativa foi realizada através da medição do diâmetro, na região mediana da célula, e do comprimento, incluindo apêndices quando presentes, dos elementos de vaso para cada órgão a partir do material macerado, utilizando o microscópio Zeiss Standard 25 e o software Zeiss KS100 3.0. Para todas as amostras foi realizada uma medição de trinta elementos de vaso e, com base nestas medições, calculado o $\mathrm{N}$ para um erro menor ou igual a $10 \%$. Quando necessário, foram então tomadas novas medidas de elementos de vaso para se obter um N satisfatório. Desta forma, o N não foi fixo e igual para todas as amostras trabalhadas, sendo fixo o erro de menor ou igual a $10 \%$.

Comparações entre diâmetro radial e tangencial foram feitas através do teste de Student's t e entre os tamanhos dos elementos de vaso das diferentes espécies através dos testes Anova e Tukey HSD, todos com $\alpha=0,05$. Análises estatísticas foram realizadas com a ajuda do software JMP 5.0.

Desenhos esquemáticos dos elementos de vaso foram realizados com o auxílio de microscópio acoplado à câmara clara. As fotomicrografias foram obtidas a partir do Sistema de Digitalização de Imagem IM50 acoplado ao microscópio Leica DMLB.

\section{Resultados e discussão}

Os elementos de vaso das espécies analisadas são predominantemente pequenos e todos com placa de perfuração simples, o que está de acordo com a descrição da família dada por Metcalfe \& Chalk (1972). A placa de perfuração varia quanto à posição de oblíqua a transversa e à localização de terminal a lateral (Fig. 1-4), sendo muito reduzida a freqüência do último tipo (Tab. 1).

Em todas as espécies foi observado que os elementos de vaso da raiz apresentam predominantemente espessamento do tipo pontoado (Fig. 5 e 6), não sendo encontrados elementos com espessamento do tipo helicoidal ou escalariforme, exceto em $P$. mucronata e $P$. werdermannii que apresentam elementos com espessamento escalariforme em uma proporção muito reduzida (Tab. 1). Por sua vez, tanto em folhas quanto no caule, o tipo de espessamento encontra-se melhor distribuído, sendo também possível observar elementos com espessamento helicoidal, escalariforme e reticulado (Fig.7-10). 
Devido ao diâmetro extremamente pequeno de grande parte dos elementos de vaso $(<25 \mu \mathrm{m}$, ver Tab. 2), segundo classificação proposta por Metcalfe \& Chalk (1985), às vezes torna-se difícil determinar a presença de aréola e a disposição alterna ou oposta das pontoações. No caso das espécies analisadas neste trabalho, esta disposição apresenta-se predominantemente
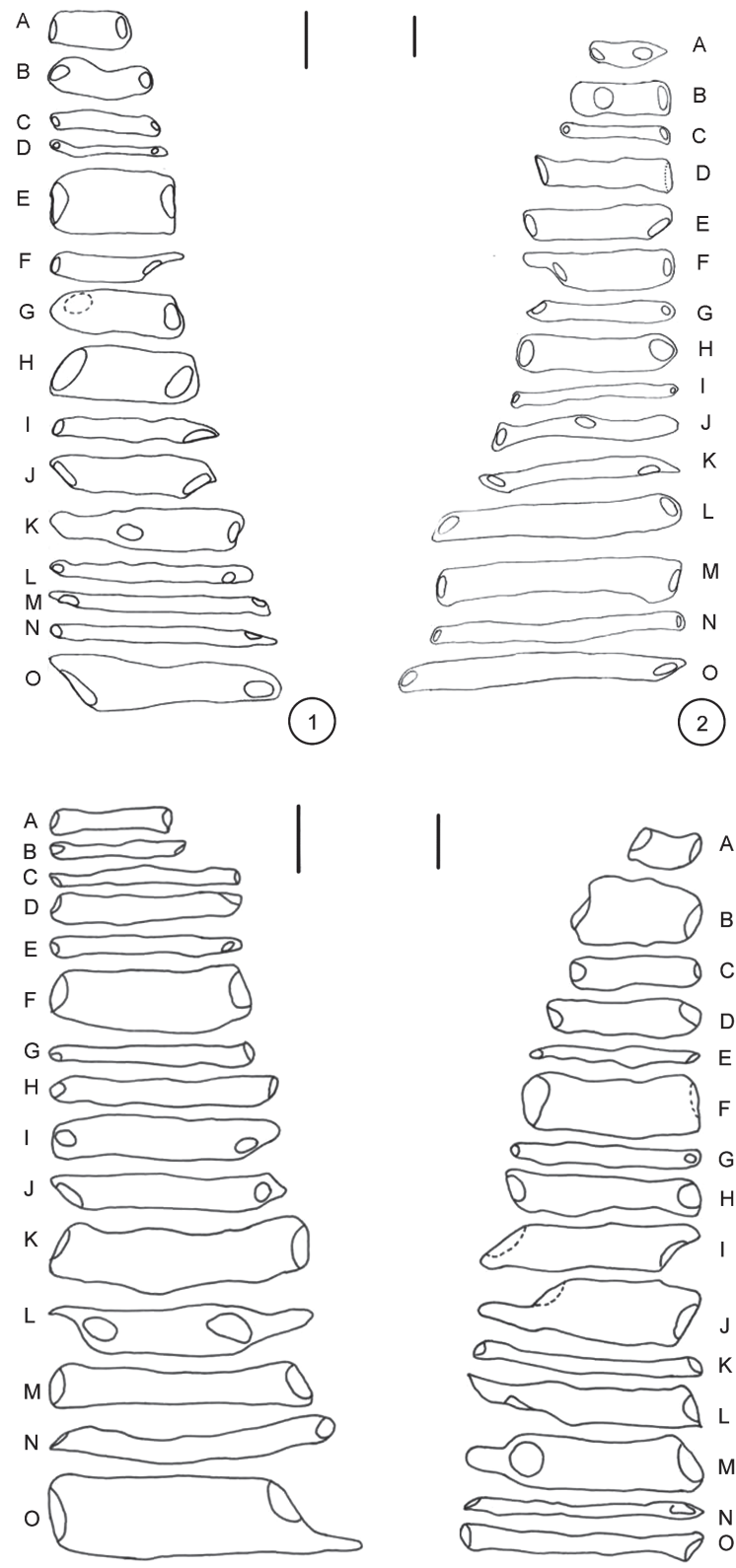

(3)

(4)

Figuras 1-4. Representação esquemática dos elementos de vaso encontrados nos órgãos vegetativos das quatro espécies de Portulaca. 1. Portulaca hirsutissima Camb. (raiz $=\mathrm{A}, \mathrm{E}, \mathrm{G}, \mathrm{I}, \mathrm{J}, \mathrm{O}$; caule $=\mathrm{B}, \mathrm{C}$, F, H, K; folha = D, L, M, N). 2. Portulaca halimoides $\mathrm{L}$. (raiz $=\mathrm{A}$, E, F, H, L, K, O; caule $=\mathrm{B}, \mathrm{D}, \mathrm{J}, \mathrm{M}$; folha $=\mathrm{C}, \mathrm{G}, \mathrm{I}, \mathrm{N})$. 3. Portulaca werdermannii Poelln. (raiz $=\mathrm{F}, \mathrm{I}, \mathrm{L}, \mathrm{M}, \mathrm{O}$; caule $=\mathrm{D}, \mathrm{E}, \mathrm{J}, \mathrm{K}, \mathrm{N}$; folha $=$ A, B, C, G, H). 4. Portulaca mucronata Link. (raiz = B, I, J, $\mathrm{L}, \mathrm{M}$; caule $=\mathrm{A}, \mathrm{D}, \mathrm{F}, \mathrm{H}, \mathrm{K} ;$ folha $=\mathrm{C}, \mathrm{E}, \mathrm{G}, \mathrm{N}, \mathrm{O})$. Barras $=50 \mu \mathrm{m}$.
Tabela 1. Dados qualitativos dos elementos de vaso de Portulaca hirsutissima Camb., Portulaca halimoides L., Portulaca mucronata Link. e de Portulaca wedermannii Poelln.

\begin{tabular}{ccc}
\hline Raiz & Caule & Folha \\
$(\%)$ & $(\%)$ & $(\%)$ \\
\hline
\end{tabular}

Portulaca hirsutissima

Espessamento da parede secundária Helicoidal

Escalariforme

Reticulado

Pontoado

Apêndice

Ambas terminações

Uma terminação

Ausente

Placa de perfuração

Ambas terminais

Ambas laterais

Terminal/Lateral

Disposição da placa

Ambas obliquas

Ambas transversas

Transversa/Obliqua

Portulaca halimoides

Espessamento da parede secundária

Helicoidal

Escalariforme

Reticulado

Pontoado

Apêndice

Ambas terminações

Uma terminação

Ausente

Placa de perfuração

Ambas terminais

Ambas laterais

Terminal/Lateral

Disposição da placa

Ambas obliquas

Ambas transversas

Transversa/Obliqua

Portulaca mucronata

Espessamento da parede secundária

Helicoidal

Escalariforme

Reticulado

Pontoado

$\begin{array}{rrr}0 & 5 & 5 \\ 0 & 8 & 10 \\ 28 & 41 & 26 \\ 72 & 46 & 59\end{array}$

$\begin{array}{lll}3 & 11 & 5\end{array}$

$\begin{array}{lll}16 & 27 & 17\end{array}$

$\begin{array}{lll}81 & 62 & 78\end{array}$

81

1

18

59

13

28

80

66

1

19

Apêndice

Ambas terminações

Uma terminação

Ausente

Placa de perfuração

Ambas terminais

Ambas laterais

Terminal/Lateral

Disposição da placa

Ambas obliquas

Ambas transversas

Transversa/Obliqua

Portulaca wedermannii

Espessamento da parede secundária Helicoidal

$\begin{array}{rrr}0 & 5 & 4 \\ 0 & 2 & 7 \\ 18 & 29 & 20 \\ 82 & 64 & 69 \\ & & \\ 8 & 13 & 3 \\ 49 & 37 & 23 \\ 43 & 50 & 74 \\ & & \\ 81 & 88 & 81 \\ 2 & 1 & 3 \\ 17 & 11 & 16 \\ & & \\ 53 & 44 & 18 \\ 24 & 18 & 48 \\ 23 & 38 & 34\end{array}$

$\begin{array}{lll}0 & 2 & 7\end{array}$

$\begin{array}{lll}1 & 32 & 34\end{array}$

$\begin{array}{lll}46 & 61 & 32\end{array}$

$\begin{array}{lll}53 & 5\end{array}$

$20 \quad 2 \quad 2$

$\begin{array}{lll}40 & 14 & 23\end{array}$

$\begin{array}{lll}40 & 84 & 75\end{array}$

$90 \quad 91 \quad 80$

$2-1$

$\begin{array}{lll}8 & 8 & 18\end{array}$

$\begin{array}{lll}52 & 29 & 35\end{array}$

$\begin{array}{lll}11 & 34 & 25\end{array}$

$\begin{array}{lll}37 & 37 & 40\end{array}$

5

10

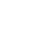

(1)

8

6

9


Tabela 1 (continuação)

\begin{tabular}{lrrr}
\hline & $\begin{array}{r}\text { Raiz } \\
(\%)\end{array}$ & $\begin{array}{c}\text { Caule } \\
(\%)\end{array}$ & $\begin{array}{c}\text { Folha } \\
(\%)\end{array}$ \\
\hline $\begin{array}{l}\text { Escalariforme } \\
\text { Reticulado }\end{array}$ & 2 & 55 & 24 \\
$\quad \begin{array}{l}\text { Pontoado } \\
\text { Apêndice }\end{array}$ & 59 & 4 & 61 \\
$\quad$ Ambas terminações & 8 & 1 & 4 \\
Uma terminação & 36 & 12 & 34 \\
$\quad$ Ausente & 56 & 87 & 62 \\
Placa de perfuração & & & \\
$\quad$ Ambas terminais & 94 & 90 & 89 \\
Ambas laterais & 0 & 1 & 2 \\
Terminal/Lateral & 6 & 9 & 9 \\
Disposição da placa & & & \\
Ambas obliquas & 52 & 34 & 41 \\
Ambas transversas & 20 & 30 & 13 \\
Transversa/Obliqua & 28 & 36 & 46 \\
\hline
\end{tabular}

desorganizada, não formando nenhum padrão específico $\mathrm{e}$, entre as formas organizadas, predomina a alterna.

Apêndices, quando presentes, podem se situar em uma ou ambas as paredes terminais do elemento de vaso e exibem o mesmo padrão de espessamento de parede secundária existente no restante da célula (Fig. 6). Não foi observada variação quanto à forma do apêndice, sendo apenas evidente diferenças no tamanho dos mesmos.

Uma análise preliminar dos diâmetros dos elementos de vaso de caule e raiz foi realizada com o intuito de testar a igualdade dos diâmetros radiais e tangenciais. $\mathrm{O}$ teste Student's t mostrou que não existe variação significativa entre o diâmetro radial e tangencial em caule e raiz das espécies estudadas, a exceção da raiz de $P$. halimoides que apresentou um diâmetro tangencial significativamente maior que o radial.

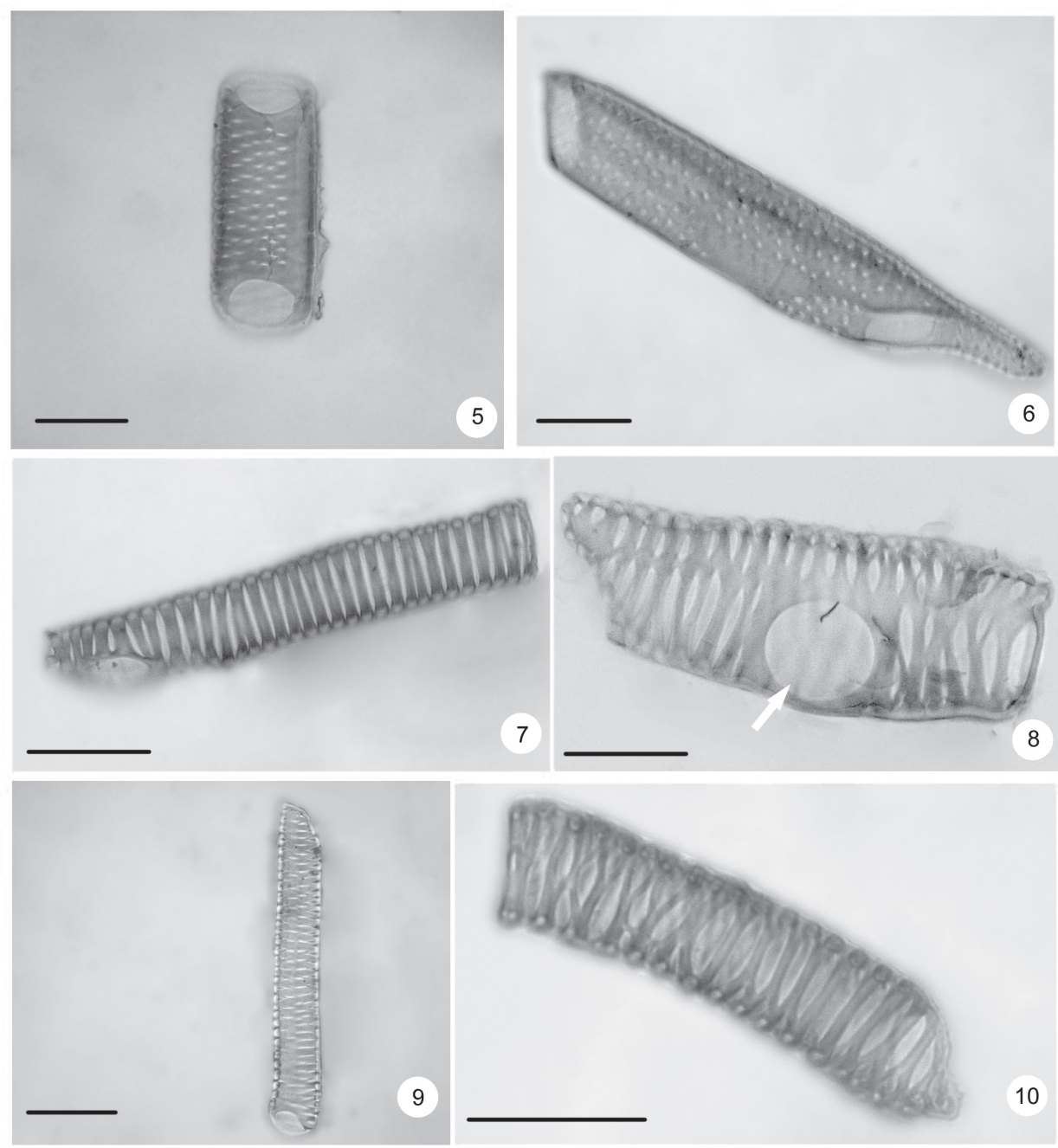

Figuras 5-10. Elementos de vaso (EV) obtidos a partir de material dissociado. 5. Elemento de vaso da raiz de Portulaca werdermannii Poelln. apresentando espessamento do tipo pontoado. 6. Elemento de vaso da raiz de Portulaca halimoides L. Note apêndice em uma das extremidades. 7. Elemento de vaso do caule de Portulaca halimoides L. com espessamento de parede secundária escalariforme. 8. Elemento de vaso da folha de Portulaca halimoides L. com placa de perfuração lateral (seta). 9. Elemento de vaso do caule de Portulaca mucronata Link. com espessamento do tipo reticulado. 10. Elemento de vaso da folha de Portulaca hirsutissima Camb. com espessamento de parede secundária reticulado. Barras $=40 \mu \mathrm{m}$. 
Tabela 2. Comprimento e diâmetro, com respectivos desvios padrões, dos elementos de vaso dos órgãos vegetativos de três indivíduos de Portulaca hirsutissima Camb., Portulaca halimoides L., Portulaca mucronata Link. e de Portulaca wedermannii Poelln., dados em $\mu$ m.

\begin{tabular}{|c|c|c|c|c|c|c|}
\hline \multirow[t]{2}{*}{ Indivíduo } & \multicolumn{2}{|c|}{1} & \multicolumn{2}{|c|}{2} & \multicolumn{2}{|c|}{3} \\
\hline & Comprimento & Diâmetro & Comprimento & Diâmetro & Comprimento & Diâmetro \\
\hline \multicolumn{7}{|c|}{ Portulaca hirsutissima } \\
\hline Caule & $117,63 \pm 36,38$ & $20,23 \pm 7,56$ & $126,18 \pm 41,49$ & $21,61 \pm 7,05$ & $129,93 \pm 35,73$ & $24,58 \pm 8,34$ \\
\hline Raiz & $116,32 \pm 29,79$ & $28,46 \pm 7,97$ & $137,57 \pm 41,03$ & $29,19 \pm 9,08$ & $139,14 \pm 35,23$ & $31,91 \pm 11,04$ \\
\hline Folha & $113,12 \pm 52,36$ & $10,33 \pm 2,16$ & $144,79 \pm 65,19$ & $10,67 \pm 2,73$ & $159,96 \pm 60,53$ & $12,19 \pm 2,64$ \\
\hline \multicolumn{7}{|c|}{ Portulaca halimoides } \\
\hline Caule & $122,22 \pm 36,53$ & $19,34 \pm 4,47$ & $113,49 \pm 32,65$ & $19,30 \pm 5,56$ & $118,16 \pm 36,04$ & $18,77 \pm 5,18$ \\
\hline Raiz & $128,15 \pm 28,44$ & $25,20 \pm 6,75$ & $118,90 \pm 38,53$ & $20,26 \pm 7,13$ & $125,05 \pm 34,60$ & $24,31 \pm 7,07$ \\
\hline Folha & $98,02 \pm 30,32$ & $12,45 \pm 3,20$ & $116,28 \pm 47,19$ & $13,36 \pm 4,90$ & $111,53 \pm 31,09$ & $10,50 \pm 1,97$ \\
\hline \multicolumn{7}{|c|}{ Portulaca mисronata } \\
\hline Caule & $127,69 \pm 8,05$ & $20,97 \pm 5,07$ & $120,87 \pm 21,81$ & $28,19 \pm 7,82$ & $132,53 \pm 35,12$ & $26,87 \pm 7,59$ \\
\hline Raiz & $141,69 \pm 31,77$ & $29,10 \pm 9,02$ & $148,69 \pm 37,03$ & $28,24 \pm 7,87$ & $137,10 \pm 3,73$ & $30,96 \pm 9,02$ \\
\hline Folha & $140,80 \pm 40,23$ & $14,97 \pm 3,65$ & $125,01 \pm 38,18$ & $14,99 \pm 3,46$ & $112,42 \pm 29,13$ & $16,48 \pm 3,73$ \\
\hline \multicolumn{7}{|c|}{ Portulaca wedermannii } \\
\hline Caule & $153,78 \pm 58,85$ & $20,78 \pm 5,10$ & $139,12 \pm 39,64$ & $25,16 \pm 5,24$ & $158,68 \pm 42,49$ & $27,63 \pm 8,22$ \\
\hline Raiz & $163,22 \pm 41,35$ & $26,90 \pm 10,58$ & $168,52 \pm 42,77$ & $46,94 \pm 17,50$ & $181,08 \pm 9,50$ & $37,92 \pm 16,05$ \\
\hline Folha & $149,44 \pm 42,72$ & $12,77 \pm 2,88$ & $160,04 \pm 44,02$ & $13,38 \pm 3,14$ & $208,03 \pm 3,25$ & $16,08 \pm 3,20$ \\
\hline
\end{tabular}

Este teste não foi aplicado às folhas uma vez que o sistema vascular destas é extremamente reduzido, tornando difícil sua análise em corte transversal. A média do diâmetro e do comprimento dos elementos de vaso de cada amostra, com seus respectivos desvios padrões pode ser observada na Tab. 2.

Pôde-se notar que nas quatro espécies analisadas, a média do diâmetro dos elementos de vaso apresenta grande diferença entre os órgãos. Os testes Anova e Tukey HSD mostraram que esta variação foi significativa para todas as espécies entre todos os órgãos, sendo os maiores valores registrados em raiz e os menores em folha (Fig. 11).

Já a média do comprimento dos elementos de vaso apresenta uma menor diferença entre os órgãos, seguindo o mesmo padrão do diâmetro em Portulaca halimoides e $P$. mucronata, enquanto que em $P$. hirsutissima e $P$. werdermannii a ordem, de maior para menor, é folha, raiz e caule (Fig. 11). O teste Anova mostrou que não existe diferença significativa entre os órgãos de P. hirsutissima. O teste Tukey HSD discrimina folha de caule e raiz em $P$. halimoides, raiz de caule e folha em $P$. mucronata e caule de folha e raiz em $P$. werdermannii.

As médias dos elementos de vaso também possuem diferenças significativas se comparadas entre as diferentes espécies. Entretanto, nem todas as amostras foram significativamente diferentes, como pode ser observado na Tab. 3.

Portulaca werdermannii foi a espécie que apresentou maiores médias de diâmetro e comprimento (Tab. 2), exceto pelo diâmetro no caule e na folha. Contudo esta diferença não foi significativa e pode-se considerar que tenha apresentado os maiores valores para estes órgãos juntamente com $P$. mucronata.

Como aponta Bailey (1957), as generalizações baseadas em métodos estatísticos tendem a obscurecer as tendências evolutivas do xilema dentro de um determinado grupo, podendo as variações qualitativas e quantitativas serem influenciadas por diversos fatores, como o habitat, idade da planta e o hábito da espécie. As espécies aqui tratadas são herbáceas, com reduzido crescimento secundário e em todas as amostras utilizadas o material apresentava apenas elementos de vasos formados pela atividade do câmbio de origem procambial, estando o câmbio de origem pericíclica produzindo apenas fibras. O ambiente é muito similar onde essas espécies ocorrem, sendo caracterizado por uma vegetação típica de campos rupestres, com solo pedregoso e algumas populações ocorrendo em bancos de areia.

Alguns caracteres do lenho são descritos na literatura como plesiomorfias, ou seja, diversificaram primeiro na história evolutiva das plantas, aparecendo nos ancestrais comuns de grandes grupos, e ocorrendo hoje em dia em grupos filogeneticamente basais. Alguns desses caracteres são listados por Carlquist (1961) como placa de perfuração escalariforme, espessamento de parede lateral escalariforme, grande comprimento com diâmetro relativamente estreito e paredes terminais oblíquas. Embora tenham sido observados alguns desses caracteres nas espécies estudadas, não os podemos considerar como plesiomorfia, pois Portulacaceae assim como outras famílias de plantas suculentas apresentam alto grau de especialização do xilema, descrito por Carlquist (1962) como pedomorfose. Este fenômeno é 

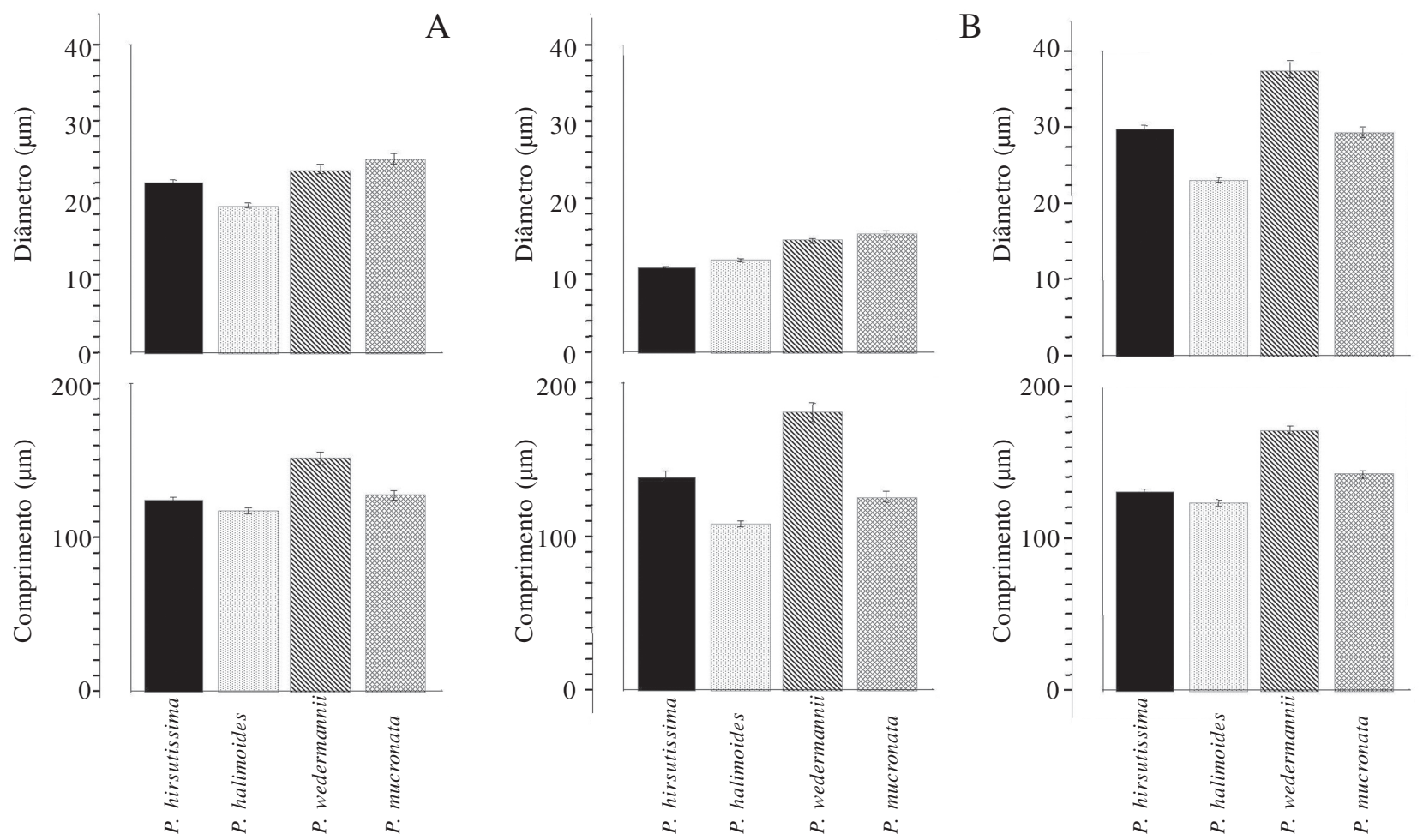

Figura 11A-C. Médias do diâmetro e comprimento dos elementos de vaso de Portulaca halimoides L., Portulaca hirsutissima Camb., Portulaca mucronata Link. e Portulaca werdermannii Poelln. separadas por órgão. A. Caule. B. Folha. C. Raiz.

caracterizado pela ocorrência de elementos de vasos secundários com caracteres típicos do xilema primário (como o padrão de espessamento de parede secundária, tamanho dos elementos, inclinação das paredes terminais e tipo de placa de perfuração). Ainda segundo o autor, em representantes de Cactaceae, a ocorrência de pedomorfose pode ser determinada por elementos de vaso do xilema secundário com padrões de espessamento de parede secundária reticulado e/ou escalariforme. Gibson (1978) também relata a ocorrência deste fenômeno em algumas Cactaceae, e chama a atenção para a necessidade de se usar os elementos de vaso com espessamento do tipo pontoado, presentes geralmente na primeira porção de xilema secundário formado na raiz, para se estimar o verdadeiro grau de especialização nestas plantas. Desta maneira, segundo o autor, essa primeira porção de xilema secundário da raiz conserva caracteres plesiomórficos do grupo, sendo então os padrões de espessamento reticulado e escalariforme, encontrados no restante da planta, um caso reversão, e não uma evidência de um sistema vascular menos derivado. Isso pode ser visualizado no presente trabalho, onde nas raízes foi observada maior freqüência de elementos de vaso pontoados, em oposição aos elementos com espessamento reticulado e escalariforme encontrados predominantemente no caule e na folha. Os resultados obtidos neste estudo caracterizam o fenômeno da pedomorfose em Portulacaceae, demonstrando que características dos elementos traqueais em Cactaceae e Portulacaceae representam parte da estratégia adaptativa à suculência, como proposto por vários autores para Cactaceae (Carlquist 1962; Gibson 1978).

Além da pedomorfose, um outro aspecto que foi abordado no presente estudo é a ocorrência de traqueídes, principalmente em folhas de $P$. hirsutissima e de $P$. werdermannii (Fig. 12-14). Nessas espécies foi registrada a presença freqüente de traqueídes que se assemelham bastante, morfologicamente, às denominadas traqueídes terminais que ocorrem nas terminações vasculares de folhas de diversas espécies de Magnoliaceae (Tucker 1964) e também aos idioblastos traqueoidais ocorrentes em Orchidaceae (Olatunji \& Nengim 1980). Segundo os autores, esses elementos traqueoidais ocorrem em diferentes órgãos dependendo da espécie, mas somente em plantas epífitas, relacionando tais células com uma possível função de adaptação ao estresse hídrico. Apesar de possuírem aspecto semelhante, estes dois tipos celulares não são comparáveis ontogeneticamente, visto que os idioblastos traqueoidais das Orchidaceae e de outras angiospermas têm origem do sistema fundamental enquanto as traqueídes terminais têm origem do sistema vascular. Do material analisado no presente trabalho, as traqueídes observadas ocorrem no sistema vascular, portanto não se trata de idioblastos traqueoidais. 
Tabela 3. Resultado do teste Tukey HSD para verificação de diferenças significativas de comprimento e diâmetro dos elementos de vaso entre as espécies. Média correspondente ao logaritmo das medias. Pares de amostras não apresentando a mesma letra são significativamente distintas.

\begin{tabular}{|c|c|c|c|c|}
\hline & Espécie & \multicolumn{3}{|c|}{ Média (Log) } \\
\hline \multicolumn{5}{|l|}{ Comprimento } \\
\hline \multicolumn{5}{|l|}{ Caule } \\
\hline P. hirsutissima & 4,77 & & B & $\mathrm{C}$ \\
\hline P. halimoides & 4,72 & & & $\mathrm{C}$ \\
\hline P. mucronata & 4,81 & & B & \\
\hline P. wedermannii & 4,97 & A & & \\
\hline \multicolumn{5}{|l|}{ Raiz } \\
\hline P. hirsutissima & 4,83 & & & $\mathrm{C}$ \\
\hline P. halimoides & 4,78 & & & $\mathrm{C}$ \\
\hline P. mucronata & 4,93 & & $\mathrm{~B}$ & \\
\hline P. wedermannii & 5,11 & A & & \\
\hline \multicolumn{5}{|l|}{ Folha } \\
\hline P. hirsutissima & 4,83 & & $\mathrm{~B}$ & \\
\hline P. halimoides & 4,63 & & & $\mathrm{C}$ \\
\hline P. mucronata & 4,79 & & $\mathrm{~B}$ & \\
\hline P. wedermannii & 5,13 & A & & \\
\hline \multicolumn{5}{|l|}{ Diâmetro } \\
\hline \multicolumn{5}{|l|}{ Caule } \\
\hline P. hirsutissima & 3,03 & & B & \\
\hline P. halimoides & 2,91 & & & $\mathrm{C}$ \\
\hline P. mucronata & 3,18 & $\mathrm{~A}$ & & \\
\hline P. wedermannii & 3,13 & A & & \\
\hline \multicolumn{5}{|l|}{ Raiz } \\
\hline P. hirsutissima & 3,34 & & B & \\
\hline P. halimoides & 3,09 & & & $\mathrm{C}$ \\
\hline P. mucronata & 3,34 & & $\mathrm{~B}$ & \\
\hline P. wedermannii & 3,52 & A & & \\
\hline \multicolumn{5}{|l|}{ Folha } \\
\hline P. hirsutissima & 2,37 & & & $\mathrm{C}$ \\
\hline P. halimoides & 2,45 & & B & \\
\hline P. mucronata & 2,71 & $\mathrm{~A}$ & & \\
\hline P. wedermannii & 2,65 & $\mathrm{~A}$ & & \\
\hline
\end{tabular}

\section{Agradecimentos}

Os Autores agradecem à Fundação de Amparo à Pesquisa do Estado de São Paulo (FAPESP), pela bolsa de Iniciação Cientifica (FAPESP 05/55072-0) concedida ao primeiro autor e pelo financiamento dado ao projeto de pesquisa (FAPESP 2004/05433-0); à Dra. Alexa A.O.P. Coelho (UEFS), pela identificação do material botânico e ao Dr. Gregório C.T. Ceccantini (IB-USP), pelas sugestões dadas na parte de estatística.

\section{Referências bibliográficas}

Arruda, E.C.; Alves, M. \& Melo-de-Pinna, G.F.A. 2004. Elementos traqueais de cinco táxons de Cactaceae da caatinga pernambucana, Brasil. Acta Botanica Brasilica 18: 731-736.

Bailey, I.W. 1957. The potentialities and limitations of wood anatomy in the phylogeny and classification of angiosperms. Journal of the Arnold Arboretum 38: 243-254.

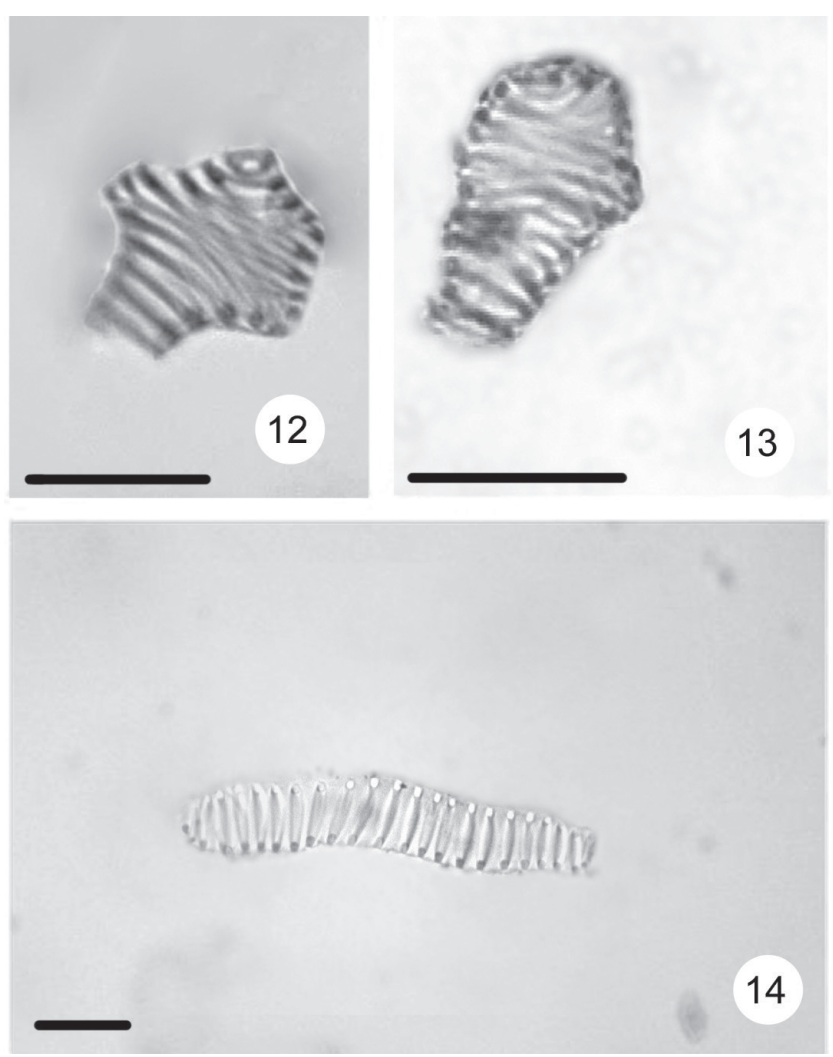

Figuras 12-14. Traqueídes vasculares obtidas a partir de material dissociado da folha de Portulaca hirsutissima Camb. e Portulaca werdermannii Poelln. 12. Portulaca hirsutissima Camb. 13-14. Tipos diferentes de traqueides encontradas em Portulaca werdermannii Poelln. Barras $=20 \mu \mathrm{m}$.

Carlquist, S. 1961. Comparative plant anatomy. New York, Holt, Rinehart and Winston.

Carlquist, S. 1962. A theory of paedomorphosis in dicotyledonous woods. Phytomorphology 12: 30-45.

Franklin, G.L. 1945. Preparation of thin sections of synthetic resins and wood-resin composites, and a new macerating method for wood. Nature 155: 51.

Gibson, A.C. 1978. Woody anatomy of plantyopuntias. Aliso 9: 279-307.

Gibson, A.C. 1994. Vascular Tissues. Pp. 45-74. In: H.D. Behnke \& T.J. Mabry (eds.). Caryophyllales: Evolution and Systematics. Berlin, Springer-Verlag.

IAWA COMMITTEE. 1989. Committee of the International Association of Wood Anatomists. List of microscopic features for hardwood identification. IAWA Bulletin 10: 219-332.

Johansen, D. 1940. Plant Microt. New York, McGraw.

Metcalfe, C.R. \& Chalk, L. 1972. Anatomy of the Dicotyledons: leaves, stem, and wood in relation to taxonomy with notes on economic uses. v.I. Oxford, Clarendon Press.

Metcalfe, C.R. \& Chalk, L. 1985. Anatomy of the Dicotyledons: wood structure and conclusion of the general introduction. Second edition. v.II. Oxford, Clarendon Press.

Olatunji, O.A. \& Nengim, R.O. 1980. Occurrence and distribution of tracheoidal elements in the Orchidaceae. Botanical Journal of the Linnean Society 80: 357-370.

Purvis, M.; Collier, D. \& Walls, D. 1964. Laboratory techniques in botany. London, Butterworths.

Souza, V. C. \& Lorenzi, H. 2005. Botânica Sistemática: guia ilustrado para identificação das famílias de Angiospermas da flora brasileira, baseado em APGII. Nova Odessa, Instituto Plantarum.

Tucker, S.C. 1964. The terminal idioblasts in magnoliaceous leaves. American Journal of Botany 51: 1051-1062.

Versão eletrônica do artigo em www.scielo.br/abb e http://www.botanica.org.br/acta/ojs 